\title{
The effect of auditory binaural beat stimulation on sustained attention
}

\author{
Matthew K. Robison ${ }^{1}$, Mohitha Obulasetty ${ }^{2}$, Chris Blais ${ }^{3}$, Kimberly \\ M. Wingert ${ }^{4}$, \& Gene A. Brewer ${ }^{3}$ \\ ${ }^{1}$ University of Texas at Arlington \\ ${ }^{2}$ Kansas City University of Medicine and Biosciences \\ 3 Arizona State University \\ ${ }^{4}$ Consortium of Universities of the Washington Metropolitan Area
}

\begin{abstract}
Binaural beats have been used as a way of modifying cognition via auditory stimulation. A recent meta-analysis suggests that binaural beat stimulation can have a positive effect on attention (Garcia-Argibay, Santed, \& Reales, 2019), with the sample-weighted average effect size being about .58. This is an intriguing and potentially useful finding, both theoretically and practically. In the present study, we focus on sustained attention. We delivered beta-frequency $(16 \mathrm{~Hz})$ binaural auditory beat stimulation during a sustained attention task (the psychomotor vigilance task). In Experiment 1, reaction times were numerically faster under beat stimulation than control stimulation in a between-subjects design. However, the effect was modest in magnitude, and model comparisons using Bayes Factors were indiscriminate between including and excluding the effect from the model. We followed this initial experiment two additional experiments. In the second experiment, we added thought probes to measure any changes in task-engagement associated with binaural beat stimulation. The beat stimulation had no effect on reaction times in Experiment 2, and it did not affect the thought probes responses. Combining data across the two experiments indicated rather strong evidence against the hypothesis that beta-frequency binaural beats can augment sustained attention. Finally, in Experiment 3, we investigated whether pupillary measures of arousal and/or task-engagement would be affected by binaural beat stimulation. There was no evidence for such effects. Overall, we did not observe any convincing evidence that binaural auditory beat stimulation effects sustained attention or its subjective and physiological correlates.
\end{abstract}

Keywords: binaural beats; sustained attention; vigilance; mind-wandering; pupillometry

Word count: 6240 
Sustaining one's attention can be quite difficult. As people complete a given task for an extended period of time, their performance tends to deteriorate. This effect is often referred to as the vigilance decrement. The precise reasons for this effect are still under debate, but recent attempts have been made to offset such effects with various interventions (Esterman et al., 2016; Esterman, Reagan, Liu, Turner, \& DeGutis, 2014; Hopstaken et al., 2015a, 2015b; Hopstaken, Linden, Bakker, Kompier, \& Leung, 2016; Massar, Lim, Sasmita, \& Chee, 2016; Matthew K Robison et al., 2020b). In a separate line of work, researchers have been employing binaural auditory beat stimulation to augment cognition, and specifically to improve attention (Hommel, Sellaro, Fischer, Borg, \& Colzato, 2016; Lane, Kasian, Owens, \& Marsh, 1998; Reedijk, Bolders, Colzato, \& Hommel, 2015; Solcà, Mottaz, \& Guggisberg, 2016). Here, we combine these approaches by examining whether binaural beat stimulation can be used to improve sustained attention. Such a finding would be both practically and theoretically meaningful. From a pragmatic perspective, binaural beats could be employed as a means of improving cognitive performance in environments where sustained attention failures could be harmful. From a theoretical perspective, augmenting sustained attention with binaural beats could provide a means for testing mechanistic explanations of why vigilance decrements occur.

Although theories differ in the precise mechanism by which it occurs, most theories of sustained attention agree that as time-on-task increases, engagement with a task decreases. The primary source of discrepancy is whether this is because participants can't continue to engage with the task (e.g., due to fatigue, resource depletion; Warm, Parasuraman, \& Matthews, 2008) or whether they choose not to (e.g., due to shifts in motivation or cost/benefit decisions; Kurzban, Duckworth, Kable, \& Myers, 2013). Powerful means of testing such theories are experimental manipulations that affect the psychology of sustained attention, without any exogenous physiological input (e.g., caffeine, pharmaceuticals), as well as manipulations that may affect physiology without systematically affecting the psychological framework of a task. Binaural beats may fall into this latter category. If binaural beats can mitigate vigilance decrements and actually augment sustained attention, they can be leveraged to disentangle competing predictions made by motivational and physiological accounts of the vigilance decrement.

Binaural beats are produced when two different tones are played into the two ears, leading to the perception of a "beat" at a frequency equal to the difference between the

Matthew K. Robison, Department of Psychology, University of Texas at Arlington; Mohitha Obulasetty, College of Osteopathic Medicine, Kansas City University of Medicine and Biosciences; Chris Blais, Department of Psychology, Arizona State University; Kimberly M. Wingert, Consortium Research Fellows Program, Consortium of Universities of the Washington Metropolitan Area; Gene A. Brewer, Department of Psychology, Arizona State University.

The data from Experiment 1 were collected as part of an Honors Thesis completed by author M.O. for the Barrett Honors College at Arizona State University. We would like to thank Stephen Goldinger for providing the eye-tracking equipment necessary to complete this project. Authors M.K.R., C.B., and G.A.B. were supported by National Science Foundation grant 1632327 during the data collection phases of this project.

Correspondence concerning this article should be addressed to Matthew K. Robison, 501 Nedderman Drive, Box 19528, Arlington, TX 76019. E-mail: matthew.robison@uta.edu 
two tones. For example, if a tone of $200 \mathrm{~Hz}$ is played into one year, and a tone of 208 $\mathrm{Hz}$ is played into the other ear, the individual will perceive an $8-\mathrm{Hz}$ beat. Prior research has demonstrated that playing these beats can augment psychological experience. A recent meta-analysis examined the effects of binaural auditory beat stimulation on anxiety, pain perception, and cognition (Garcia-Argibay, Santed, \& Reales, 2019). Overall, the metaanalysis determined an average effect size of .45 in the positive direction. That is, playing binaural beats improved cognitive performance, reduced anxiety, and reduced the intensity of pain perception. Most relevant to the current study, the meta-analysis found an average effect size of $.58(95 \%$ CI .34 - .83) on attention. There is not complete agreement on the mechanism by which binaural beats affect cognition and behavior. It may be that the beats cause phase-resetting and entrainment of neural oscillations to the beats, which can cause increases in neural gain and more optimal responding to incoming stimuli (Lakatos, Karmos, Mehta, Ulbert, \& Schroeder, 2008). It may also be that the beats increase interhemispheric coherence, as the auditory system tries to make sense of the two inputs (Solcà et al., 2016).

Most relevant to the present study is the fact that several studies have specifically shown improvements in attention with binaural beat stimulation. As a few examples, Lane et al. (1998) found an improvement in target detection during a vigilance task with betafrequency (16 and $24 \mathrm{~Hz}$ ) binaural beat stimulation compared to delta/theta-frequency beats $(1.5$ and $4 \mathrm{~Hz}$ ) stimulation. Reedijk et al. (2015) found a reduction in the attentional blink with gamma-frequency $(40 \mathrm{~Hz})$ beats compared to alpha-frequency $(10 \mathrm{~Hz})$ beats and a constant tone, but only for individuals low in preexperimental spontaneous eye-blink rate. In a task that induces a psychological refractory period, Hommel et al. (2016) showed an increased response-compatibility effect under gamma-frequency $(40 \mathrm{~Hz})$ beats compared to a constant tone. Specifically, participants who heard the binaural beat stimulus showed an increased response compatibility effect, indicating the gamma-band beats may have induced greater cognitive flexibility. However some studies have failed to demonstrate effects on behavior. For example, Solcà et al. (2016) tested the effect of alpha-frequency $(10 \mathrm{~Hz})$ stimulation on performance in a dichotic listening task. Although they did find an effect on interhemispheric coherence in EEG data, they did not find an improvement in listening performance compared to a control stimulation condition (monaural beats).

\section{The Present Study}

In the present study we focus on the effect of binaural auditory beat stimulation on sustained attention. We gave participants a 20-minute simple reaction time task while they listened to either beta-frequency $(16 \mathrm{~Hz})$ binaural beat stimulation or a pure tone control stimulus. We wanted to investigate whether binaural beats would produce general changes in task performance, mitigation of the vigilance decrement, and/or changes in subjective task-engagement.

\section{Experiment 1}

In Experiment 1, a sample of participants completed the psychomotor vigilance task

with one of two audio recordings playing through headphones. The psychomotor vigilance 
task is a simple reaction time task where participants must react as quickly as possible to the unpredictable onset of a stimulus (Dinges \& Powell, 1985). This task requires continuous attentional engagement within and across trials to maintain fast reaction times (RTs). Prior experiments with this task have consistently demonstrated a vigilance decrement which manifests as a slowing of RTs across time (Massar et al., 2016; Robison, 2018; Unsworth \& Robison, 2016, 2018, 2020). In the binaural beat condition, the auditory stimulus was created to induce a binaural beat at $16 \mathrm{~Hz}(200 \mathrm{~Hz}$ into one ear, $216 \mathrm{~Hz}$ into the other ear). In the control condition, the auditory stimulus was a constant pure tone of $200 \mathrm{~Hz}$ playing into both ears. In our analyses, we examined three potential ways that binaural beat stimulation could affect performance. In one way, the binaural beat stimulation could produce a general speeding of RTs. This would predict a main effect of binaural beat stimulation on reaction time (Figure 1a). In a second way, the binaural beat stimulation could offset the slowing of RTs across time (i.e., vigilance decrement). This would predict a beat stimulation $\mathrm{x}$ time interaction (Figure 1b). Additionally, the binaural beat stimulation could produce both a general speeding of RTs and an offsetting of the vigilance decrement (Figure 1c). These analyses provide for a more precise description of the effects binaural beat stimulation might have on sustained attention, which could lead to more specific hypothesizing about cognitive and neural mechanisms.

a)



c)

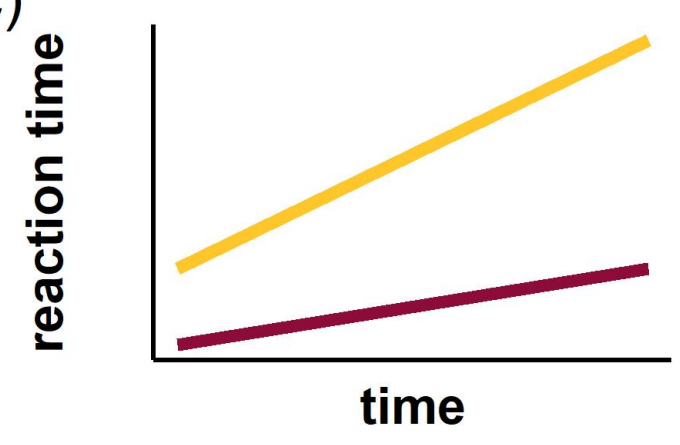

b)

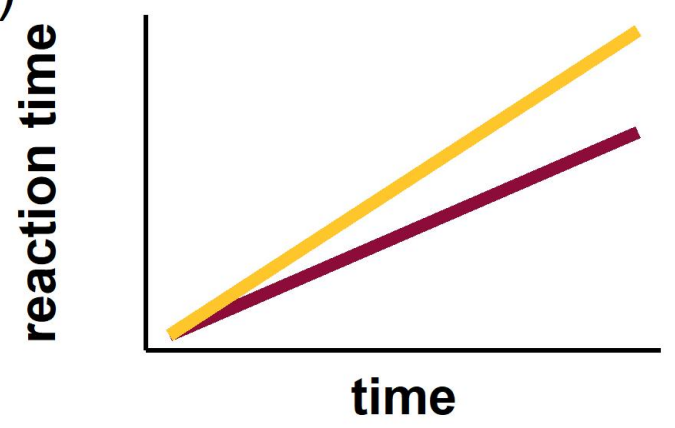

Figure 1. Hypothetical effects of binaural auditory beat stimulation on reaction times in the psychomotor vigilance task. If the stimulation does indeed affect performance, it could do so by a) a general speeding of reaction times, b) an offsetting of the vigilance decrement, or c) both a general speeding of reaction times and an offsetting of the vigilance decrement. 
Table 1

Coefficients of full mixed effect model on reaction times in Experiment 1

\begin{tabular}{lccccccc}
\hline Effect & $\beta$ & $S E$ & $t$ & $p$ & $d$ & $B F_{10}$ & $B F_{01}$ \\
\hline Block & 0.112 & 0.003 & 32.863 & $<.001$ & 0.392 & $>100,000$ & $<.01$ \\
Binaural beat & -0.097 & 0.042 & -2.310 & 0.022 & 0.348 & 1.379 & 0.725 \\
Block x Binaural beat & -0.002 & 0.005 & -0.463 & 0.643 & 0.006 & 0.023 & 44.183 \\
\hline
\end{tabular}

Note. ICC $=0.19, \beta=$ standardized regression coefficient, $S E=$ standard error of regression coefficient, $d=$ effect size, $B F_{10}=$ ratio of evidence (Bayes Factor) in favor of including effect, $B F_{01}=$ ratio of evidence (Bayes Factor) against including effect.

\section{Method}

\section{Data analysis}

We report how we determined our sample size, all data exclusions (if any), all manipulations, and all measures in the study. In all experiments, we trimmed RTs by eliminating any RT faster than $200 \mathrm{~ms}$ (anticipations) or longer than 3,000 ms. If a participant had more than 15 such trials, their data were excluded. We also excluded any outlying data point that fell more than 3 standard deviations outside its group's mean. We analyzed all data with $\mathrm{R}$ software. We used the tidyverse (Wickham, 2017) set of packages to organize and aggregate data, the lmerTest (Kuznetsova, Brockhoff, \& Christensen, 2017) and lme4 (Bates, Mächler, Bolker, \& Walker, 2015) packages to fit linear mixed effect models, the BayesFactor package (Morey \& Rouder, 2018) to compare models using Bayes Factors, and the retimes (Massidda, 2013) package to estimate ex-Gaussian parameters. The manuscript was written with the papaja (Aust \& Barth, 2018) package. All data and analysis scripts, including the $\mathrm{R}$ markdown document used to generate this manuscript, can be found on the Open Science Framework at the following URL: https://osf.io/8qfut/

We used linear mixed effects models to estimate the effects of time, binaural beat stimulation, and their interaction. Block was treated as a continuous variable in the models and was centered before the analyses. Thus, block is our time variable in the analysis. We allowed each participant's intercept to randomly vary as well. ${ }^{1}$ Thus, subject was treated as a random effect in all the models, and block and condition were treated as fixed effects. Reaction times were standardized before analysis so we could report standardized regression coefficients. To evaluate the data, we use a combination of traditional null hypothesis statistical testing with $p$ values, as well as Bayes Factors (BFs) to assess the weight of evidence in favor of different models. We considered Bayes Factors $>3$ as substantial evidence. As a final step in the analysis, we also analyzed the RTs with ex-Gaussian parameter estimates. Specifically, we extracted three parameters from the distribution of each individual's RTs: $\mu$, which represents the point of central tendency of the distribution, $\sigma$ which represents the degree of variability in the distribution, and $\tau$ which represents the length of the tail

\footnotetext{
${ }^{1}$ We attempted to allow the effect of block vary across participants as well (random slope), but some models with this specification did not converage upon a solution, so we only allowed the intercept to vary.
} 
of the distribution. In prior research with the psychomotor vigilance task, motivational incentives have been demonstrated to specifically affect $\mu$, whereas other experiments, like putting participants in acute pain have shown specific effects on $\tau$ (Matthew K Robison et al., 2020a).

\section{Participants}

A sample of 185 participants from the human subjects pool at Arizona State University completed the study in exchange for partial course credit. We had to exclude 6 participants for having more than 15 excluded RTs. The target sample size was 100 participants in each condition, but we used the end of an academic term as our stopping rule for data collection. After outlier exclusions, the final sample included 88 in the binaural beat condition and 91 in the control condition. With this sample size, we were able to detect moderate effects (Cohen's $d>=.41$ ) with $80 \%$ power. The experimental design, analysis plan, and data collection procedures were preregistered on the Open Science Framework prior to data collection (https://osf.io/xaywg).

\section{Procedure}

Participants completed a 20-minute version of the psychomotor vigilance task (Dinges $\&$ Powell, 1985). Each trial of the task began with a row of blue zeroes centered on the screen (00.000). After a random time interval sampled from a uniform distribution of 2 to $10 \mathrm{~s}$ in 500-ms steps, the zeroes began "counting up" like a stopwatch. The participant's task was to press the spacebar as quickly as possible when they noticed the numbers begin counting. After the participant pressed the spacebar, the screen displayed their RT for that trial in red font (e.g., 00.346) for $1 \mathrm{~s}$. After a 1-s blank intertrial interval, the next trial began. Participants first completed 8 practice trials. The task ran for 20 minutes (about 150 trials). After the 20-minute time limit, the task ended. We also recorded footage from a webcam while participants completed the task in order to track facial expressions across time. However, these data could not be analyzed for many participants because they moved out of range of the webcam. Therefore, they are not analyzed here.

\section{Results \& Discussion}

The data are plotted by block and condition in Figure 2. $0.17 \%$ of trials were excluded from the analysis for falling outside 3 standard deviations of their condition's mean. We tested our hypotheses with a series of linear mixed effect models. Specifically, we compared four increasingly parameterized nested models. The first model contained only a random intercept for each participant. The next model contained an effect of block. There was strong evidence supporting a model in which RTs changed across blocks. This indicates that there was a robust vigilance decrement in RTs, replicating prior research using this task (Massar et al., 2016; Massar, Lim, Sasmita, \& Chee, 2019; Robison et al., 2020b; Unsworth \& Robison, 2016, 2018, 2020). The next model contained main effects of block and condition. Here, the evidence was rather indiscriminate. That is, the data did not 
strongly favor exclusion or inclusion of a main effect of binaural beat stimulation. The fourth model contained main effects of block and condition and their interaction. This model tests whether the data reflected a systematic difference in the change in RT across blocks by condition. There was strong evidence against the inclusion of the interaction. Table 1 summarizes the full model (main effects of block, condition, and block x condition interaction). Finally, the ex-Gaussian analyses indicated that $\mu$ did not differ across conditions, $t(171)=-0.97, p=0.33, d=0.15, B F_{01}=3.93$, and nor $\operatorname{did} \sigma, t(171)=-0.35$, $p=0.73, d=0.05, B F_{01}=5.75$, or $\tau, t(171)=-0.34, p=0.73, d=0.05, B F_{01}=5.76$. Therefore we can conclude that the binaural auditory beat stimulation did not mitigate or eliminate the vigilance decrement.

Overall, the data suggested that the effect of the beta-frequency binaural beat stimulation on RTs was moderate in magnitude (0.35), but there was not strong evidence in favor of this effect $\left(B F_{10}=1.38\right)$. The estimate for the block $\mathrm{x}$ condition interaction was very small $(0.01)$, and there was strong evidence against this effect $\left(B F_{01}=44.18\right)$. Thus the data were most consistent with binaural beat stimulation causing a general speeding of responses (Figure 1a). The results are quite similar to those of Lane et al. (1998), who found a modest increase in target detection during a sequential perceptual discrimination (1-back) task with beta-band auditory beat stimulation compared to theta/delta band beat stimulation. Lane et al. did not observe an offsetting of the vigilance decrement due to beta beat stimulation.

Given the fact that the main effect was modest in magnitude, and the Bayes Factor did not heavily favor the inclusion of the effect in the model, we decided to run two additional experiments to confirm the presence of the effect and identify potential behavioral and physiological correlates of it. In Experiment 2, we largely replicated the method of Experiment 1, but we added thought probes to the task to measure subjective task engagement. In Experiment 3, we used pupillometry to investigate well-established physiological measures derived from the psychomotor vigilance task.

\section{Experiment 2}

In Experiment 2, we attempted to replicate the findings of Experiment 1, and we collected one additional dependent variable. We added thought probes to the task to measure engagement with the task. Thought probing has become a fairly common method to measure subjective attentional states as people complete tasks (Matthew K Robison et al., 2019; Weinstein, 2018). One means by which the binaural auditory beat stimulation decreased RTs may have been via increased subjective task engagement. If that is the case, we should see fewer reports of task-unrelated thoughts (TUTs) among participants in the beat condition. Other than the addition of these probes, the task parameters were largely the same. We used the same auditory stimulation for the beat and control conditions as those used in Experiment 1. 
a)

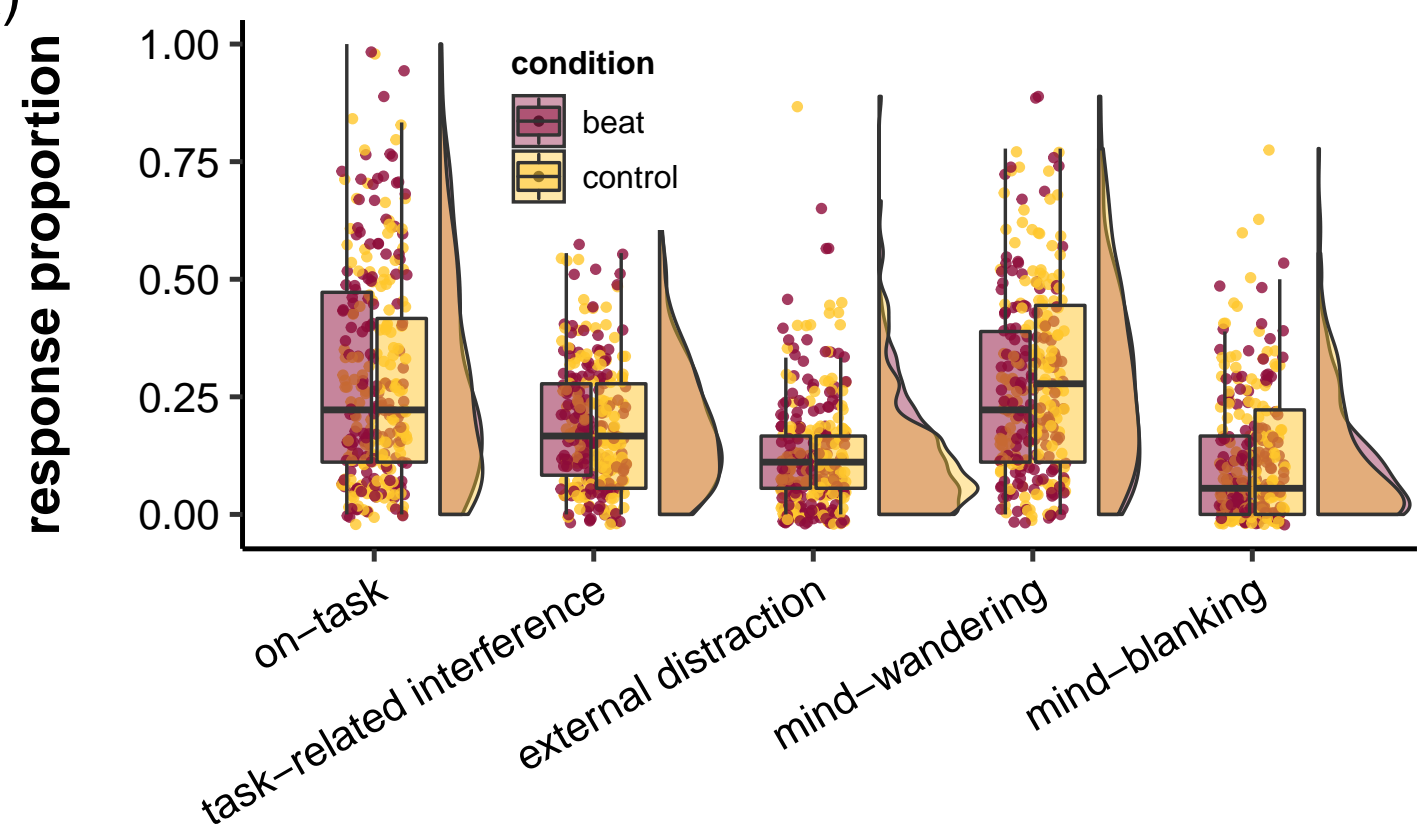

b)

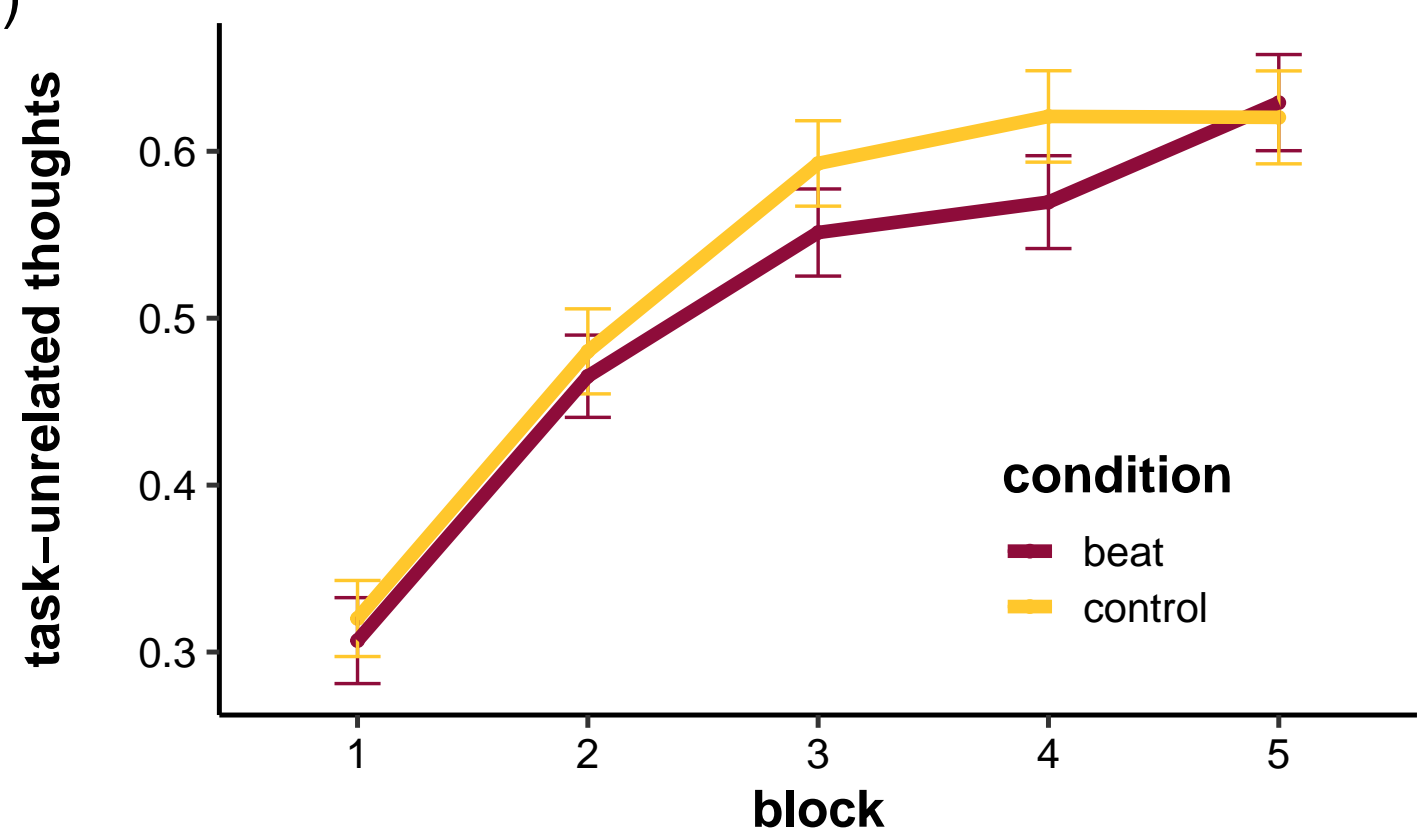

Figure 2. a) Thought probe responses by condition in Experiment 2. None of the response options significantly differed across conditions. Each data point represents the response proportion for an individual subject. Black bars represent the mean of each condition. b) Task-unrelated thoughts (TUTs) by block and condition. TUTs increased across blocks in both conditions, but did not differ across conditions. 


\section{Method}

\section{Participants and Procedure}

We specified our target sample size with a power analysis based on the between-group effect size from Experiment $1 .^{2}$ We specified an $\alpha$ of .05 and power of .80 , one-tailed. The power analysis indicated a required sample size of 296 participants (148 per condition). We used the day we reached this target sample size as our stopping rule for data collection. Before data exclusions, we ended with a sample size of 310 participants. After excluding participants for having more than 15 unusable RTs or falling more than $3 S D$ s outside their condition's mean, we ended with a final sample size of 302 participants (151 in beat condition, 151 in control condition). ${ }^{3}$ All participants were recruited through the human subjects pool at Arizona State University, gave informed consent before participating, and were compensated with partial course credit for their participation. The experimental procedure was approved by the Institutional Review Board at Arizona State University.

\section{Task}

The task was nearly identical to that used in Experiment 1 with a few exceptions. First, the task was set to run for exactly 150 trials, rather than exactly 20 minutes. The task duration was still roughly 20 minutes. We also added thought probes to the task after 18 trials spaced pseudorandomly throughout the task. ${ }^{4}$ The thought probes were screens that asked participants, "What were you thinking about in the few seconds preceding this screen?" The screen gave five options: 1) I was focused on the current task, 2) I was thinking about my performance on the task, 3) I was distracted by sights/sounds in my environment, 4) I was thinking about things unrelated to the task, and 5) My mind was blank. Response 1 was recorded as on-task, response 2 as task-related interference, and responses 3 through 5 as task-unrelated thoughts (TUTs).

\section{Results \& Discussion}

The RT data are plotted in Figure 2. As is visible, there was little separation between the two conditions. To examine this statistically, we used the same step-wise model comparison procedure as in Experiment 1 (see Table 2). There was a large effect of block on RTs. Thus we again observed a robust vigilance decrement, replicating Experiment 1 and prior research. Unlike Experiment 1, there was no effect of the binaural beat stimulation on RT. In fact, there was strong evidence against such an effect. Finally, like Experiment 1, there was strong evidence against a block x condition interaction (see Table 2). Finally, the

\footnotetext{
${ }^{2}$ Note: the reported effect size is a bit larger than the effect size we used to perform the power analysis $(d$ $=.29$ ) because we did not screen for outliers as stringently in our first analysis of the data as we did here.

${ }^{3}$ Participants were run in groups (maximum size 8 participants), and signed up for the study ahead of time. We allowed participants who had signed up before we reached our cutoff to complete the study, and we retained their data.

${ }^{4} \mathrm{~A}$ probe appeared every 16 trials. Because of the randomization of wait times, this meant a probe appeared roughly once every 100 seconds.
} 
Table 2

Coefficients of full model on task-unrelated thoughts in Experiment 2

\begin{tabular}{lccccccc}
\hline Effect & $\beta$ & $S E$ & $t$ & $p$ & $d$ & $B F_{10}$ & $B F_{01}$ \\
\hline Block & 0.091 & 0.002 & 37.394 & $<.001$ & 0.36 & $>100,000$ & $<.01$ \\
Binaural beat & 0.008 & 0.028 & 0.294 & 0.769 & 0.03 & 0.049 & 20.476 \\
Block x Binaural beat & -0.001 & 0.003 & -0.368 & 0.713 & 0.00 & 0.016 & 63.277 \\
\hline
\end{tabular}

Note. ICC $=0.17, \beta=$ standardized regression coefficient, $S E=$ standard error of regression coefficient, $d=$ effect size, $B F_{10}=$ ratio of evidence (Bayes Factor) in favor of including effect, $B F_{01}=$ ratio of evidence (Bayes Factor) against including effect.

Table 3

Coefficients of full model on task-unrelated thoughts in Experiment 2

\begin{tabular}{lccccccc}
\hline Effect & $\beta$ & $S E$ & $t$ & $p$ & $d$ & $B F_{10}$ & $B F_{01}$ \\
\hline Block & 0.074 & 0.006 & 11.493 & $<.001$ & 0.32 & $>100,000$ & $<.01$ \\
Binaural beat & 0.024 & 0.027 & 0.884 & 0.378 & 0.10 & 0.095 & 10.495 \\
Block x Binaural beat & 0.000 & 0.009 & 0.009 & 0.993 & 0.00 & 0.042 & 23.950
\end{tabular}

Note. ICC $=0.17, \beta=$ standardized regression coefficient, $S E=$ standard error of regression coefficient, $d=$ effect size, $B F_{10}=$ ratio of evidence (Bayes Factor) in favor of including effect, $B F_{01}=$ ratio of evidence (Bayes Factor) against including effect.

ex-Gaussian parameters also did not differ across conditions $(\mu: t(297)=0.06, p=0.95, d$ $=0.01, B F_{01}=7.84 ; \sigma: t(297)=0.14, p=0.89, d=0.02, B F_{01}=7.78 ; \tau: t(297)=-0.09$, $\left.p=0.93, d=0.01, B F_{01}=7.83.\right)$

Our next set of analyses focused on responses to the thought probes. Figure 3 shows the average response proportions by condition. We categorized the external distraction, mind-wandering, and mind-blanking responses as TUTs. Much in the same way we analyzed RTs, we submitted TUTs to a linear mixed effect model with fixed effects of block and condition and a random intercept for each subject. The analyses yielded similar findings to the RT analyses (see Table 3). There was a large effect of block on TUTs, such that they increased over time. This is consistent with much prior work demonstrating such an effect (McVay \& Kane, 2012; Thomson, Seli, Besner, \& Smilek, 2014; Unsworth \& Robison, 2016). However, there was strong evidence against an effect of binaural beat stimulation. Finally, there was also strong evidence against a block x condition interaction on TUTs. Therefore, we can conclude that although TUTs increased across time, they were not affected by the binaural beat stimulation, and the beat stimulation did not mitigate the effect of time on TUTs.

\section{Combined Analysis}

We combined the data across experiments and analyzed the aggregated data to examine the fact that there was an effect of beat stimulation in Experiment 1, but not in 
Table 4

Coefficients of full model on reaction times combining data across Experiments 1 and 2

\begin{tabular}{lccccccc}
\hline Effect & $\beta$ & $S E$ & $t$ & $p$ & $d$ & \multicolumn{1}{c}{$B F_{10}$} & $B F_{01}$ \\
\hline Block & 0.152 & 0.004 & 35.599 & $<.001$ & 0.271 & $>100,000$ & $<.01$ \\
Binaural beat & -0.114 & 0.055 & -2.095 & 0.037 & -0.192 & 0.142 & 7.039 \\
Experiment & -0.344 & 0.049 & -7.099 & $<.001$ & -0.650 & $>100,000$ & $<.01$ \\
Block x Binaural beat & -0.003 & 0.006 & -0.509 & 0.611 & -0.004 & 0.016 & 62.288 \\
Block x Experiment & -0.015 & 0.005 & -2.772 & 0.006 & -0.021 & 12.151 & 0.082 \\
Binaural beat x Experiment & 0.119 & 0.069 & 1.729 & 0.084 & 0.158 & 0.485 & 2.062 \\
Block x Binaural beat x Experiment & 0.002 & 0.008 & 0.277 & 0.782 & 0.002 & 0.020 & 49.673 \\
\hline
\end{tabular}

Note. $\mathrm{ICC}=0.22, \beta=$ standardized regression coefficient, $S E=$ standard error of regression coefficient, $d=$ effect size, $B F_{10}=$ ratio of evidence (Bayes Factor) in favor of including effect, $B F_{01}=$ ratio of evidence (Bayes Factor) against including effect.

Experiment 2. We specified a linear mixed effect model with fixed effects of block, condition, experiment, and a random intercept for each subject. A summary of the full model is shown in Table 4. Overall, the main effect of the binaural beat stimulation on RTs was small $(d=-0.19)$, and there was considerable evidence against including it in the model according to the Bayes Factors $\left(B F_{01}=7.04\right)$. There was also strong evidence against a block $\mathrm{x}$ condition interaction $\left(B F_{01}=62.29\right)$. Interestingly, comparing Experiment 1 to Experiment 2 revealed some unexpected findings. First, there was a large main effect of experiment $\left(d=-0.65, B F_{10}>100,000\right)$, such that RTs were significantly faster overall in Experiment 2. Second, there was evidence for a small but significant block x experiment interaction $\left(d=-0.02, B F_{10}=12.15\right)$, such that the vigilance decrement was shallower in Experiment 2. The evidence for an experiment $\mathrm{x}$ beat interaction was indiscrimate. Finally, the ex-Gaussian parameter estimates did not differ across beat and control conditions $(\mu$ : $t(473)=-0.83, p=0.41, d=0.08, B F_{01}=7.03 ; \sigma: t(473)=-1.04, p=0.30, d=0.10$, $B F_{01}=5.82 ; \tau: t(473)=-1.04, p=0.30, d=0.10, B F_{01}=5.82$.) We address potential reasons for differences across experiments in the General Discussion.

\section{Experiment 3}

In Experiment 3, we leveraged pupillometry to test whether binaural beat stimulation would increase arousal and/or increase attentional effort. In this task, pupil diameter provides two important metrics. First, we can use pupil diameter during intertrial intervals to measure moment-to-moment arousal levels. Second, the psychomotor vigilance task produces a sterotypical task-evoked pupillary response (Massar et al., 2016, 2019; Unsworth \& Robison, 2016, 2018). Thus, we can use pupillometry to test the effect of binaural beat stimulation on 1) global arousal levels, and 2) trial-to-trial changes in arousal levels, which often correlate with task performance (Matthew K Robison \& Unsworth, 2019; Unsworth \& Robison, 2017), and task-evoked pupillary responses, which are often used to index effort or attentional allocation to a task (Beatty, 1982). 


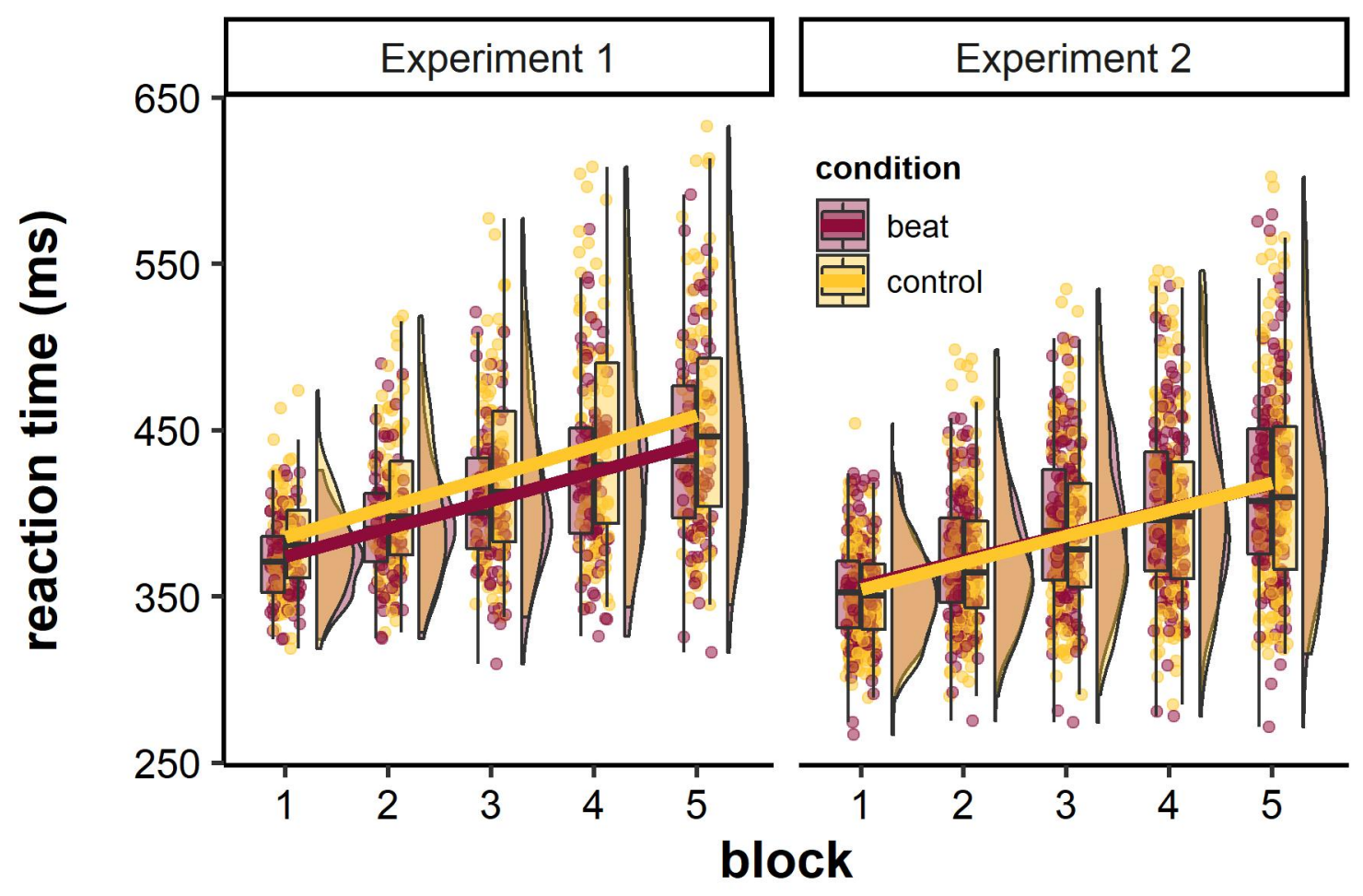

Figure 3. Reaction times by block and condition across Experiments 1 and 2. Binaural beat stimulation had a modest main effect on reaction times. Reaction times were also faster in Experiment 2 compared to Experiment 1, and there was a shallower vigilance decrement in Experiment 2. Each data point in the average reaction time in a block for an individual subject. Black bars represent the mean of each condition, and the solid lines represent the line of best fit for the effect of block in each condition

Table 5

Coefficients of full model on reaction times in Experiment 3

\begin{tabular}{lccccccc}
\hline Effect & $b$ & $S E$ & $t$ & $p$ & $d$ & $B F_{10}$ & $B F_{01}$ \\
\hline Block & 0.191 & 0.024 & 8.003 & $<.01$ & 0.142 & $>100,000$ & $<.001$ \\
Session & 0.137 & 0.060 & 2.292 & 0.022 & 0.107 & $>100,000$ & $<.001$ \\
Binaural beat & -0.053 & 0.166 & -0.319 & 0.750 & -0.016 & 3.745 & 0.267 \\
Block x Session & -0.064 & 0.015 & -4.206 & $<.01$ & -0.075 & 209.978 & 0.005 \\
Block x Binaural beat & -0.099 & 0.034 & -2.929 & $<.01$ & -0.052 & 64.240 & 0.016 \\
Session x Binaural beat & 0.068 & 0.111 & 0.611 & 0.541 & 0.030 & 1.008 & 0.992 \\
Block x Session x Binaural beat & 0.039 & 0.022 & 1.812 & 0.070 & 0.032 & 0.067 & 14.967 \\
\hline
\end{tabular}

Note. ICC $=0.25, \beta=$ standardized regression coefficient, $S E=$ standard error of regression coefficient, $d=$ effect size, $B F_{10}=$ ratio of evidence (Bayes Factor) in favor of including effect, $B F_{01}$ $=$ ratio of evidence (Bayes Factor) against including effect. 


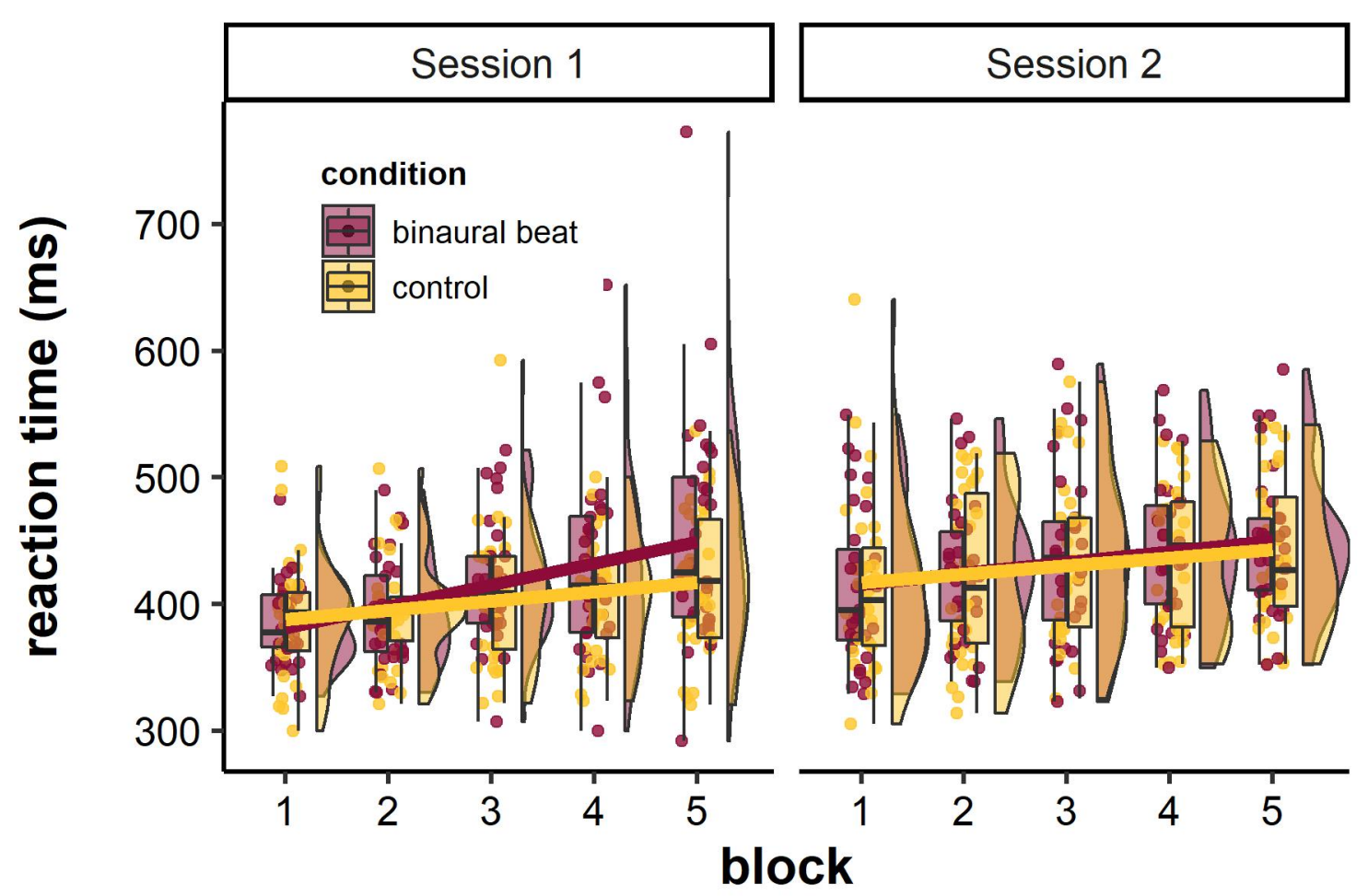

Figure 4. Reaction times by session, block, and condition in Experiment 3. Participants who heard the binaural beat stimulation in Session 1 showed a steeper vigilance decrement. There were no other differences in RTs due to the binaural auditory beat stimulation. Each data point represents the mean reaction time in a block for an individual participant. The solid lines show the group-level change in reaction time across blocks.

Table 6

Coefficients of full model on pretrial pupil diameter in Experiment 3

\begin{tabular}{lccccccl}
\hline Effect & $b$ & $S E$ & $t$ & $p$ & $d$ & $B F_{10}$ & $B F_{01}$ \\
\hline Block & 0.030 & 0.017 & 1.696 & 0.090 & 0.033 & 109.325 & 0.009 \\
Session & -0.090 & 0.212 & -0.425 & 0.672 & -0.110 & 0.159 & 6.284 \\
Binaural beat & -0.325 & 0.636 & -0.511 & 0.611 & -0.133 & 0.023 & 43.948 \\
Block x Session & -0.036 & 0.011 & -3.109 & 0.002 & -0.060 & 0.080 & 12.449 \\
Block x Binaural beat & -0.064 & 0.024 & -2.601 & 0.009 & -0.050 & 0.128 & 7.786 \\
Session x Binaural beat & 0.219 & 0.424 & 0.518 & 0.607 & 0.135 & 1.026 & 0.974 \\
Block x Session x Binaural beat & 0.051 & 0.016 & 3.204 & 0.001 & 0.062 & 7.870 & 0.127 \\
\hline
\end{tabular}

Note. ICC $=0.67, \beta=$ standardized regression coefficient, $S E=$ standard error of regression coefficient, $d=$ effect size, $B F_{10}=$ ratio of evidence (Bayes Factor) in favor of including effect, $B F_{01}=$ ratio of evidence (Bayes Factor) against including effect. 
Table 7

Coefficients of full model on task-evoked pupillary responses in Experiment 3

\begin{tabular}{lccccccc}
\hline Effect & $b$ & $S E$ & $t$ & $p$ & $d$ & $B F_{10}$ & $B F_{01}$ \\
\hline Session & -0.019 & 0.012 & -1.625 & 0.108 & -0.36 & 37.651 & 0.027 \\
Binaural beat & -0.002 & 0.033 & -0.069 & 0.945 & -0.02 & 0.241 & 4.155 \\
Session x Binaural beat & 0.002 & 0.022 & 0.073 & 0.942 & 0.02 & 0.535 & 1.870 \\
\hline
\end{tabular}

Note. ICC $=0.60, \beta=$ standardized regression coefficient, $S E=$ standard error of regression coefficient, $d=$ effect size, $B F_{10}=$ ratio of evidence (Bayes Factor) in favor of including effect, $B F_{01}=$ ratio of evidence (Bayes Factor) against including effect.

If binaural beat stimulation has an effect on arousal, it could do so in a few nonmutally exclusive ways. First, it could increase global arousal levels. If this is the case, we should see a general increase in pupil diameter during binaural beat stimulation compared to control stimulation. Second, binaural beat stimulation could also regulate arousal. If this is the case, we should see a stabilization of arousal across time. As performance tends to deteriorate across time during sustained attention tasks, pupillary measures of arousal tend to deteriorate as well (Hopstaken et al., 2015a, 2016, 2015b; Massar et al., 2016; Unsworth \& Robison, 2016, 2018). If this occurs, we should see an time $\mathrm{x}$ condition interaction (i.e., shallower drop in arousal with binaural beat stimulation). Finally, if binaural beat stimulation works to increase effort toward a task, we should see an increase in task-evoked pupillary responses compared to control stimulation conditions.

\section{Method}

A sample of participants completed the psychomotor vigilance twice within the same session: once under binaural beat stimulation and once under control stimulation. During both sessions, participants completed the study while their pupil diameters were continuously recorded via an eye-tracker. We used a within-subjects design to more tightly compare arousal and task-evoked pupillary responses across conditions. We based our sample size on a power analysis using the effect size from Experiment 1. This analysis indicated we would need a sample size of 62 participants for a within-subjects analysis to detect the effect with $80 \%$ power.

\section{Participants and Procedure}

A sample of 67 participants from the human subjects pool at Arizona State University completed the study in exchange for partial course credit. Participants first gave informed consent, then completed two rounds of the psychomotor vigilance task that each took about 20 minutes. The sessions were separated by an intervening task (the discrete whole-report visual working memory task; Adam, Mance, Fukuda, \& Vogel, 2015) that took about 15 minutes. This task was included to give participants a break from the psychomotor vigilance task, but to keep participants similarly engaged with a cognitive task. 


\section{Task}

The task was the same as that used in Experiments 1 and 2 with a few minor changes. First, there were no thought probes inserted between trials. We removed the thought probes because they may have been the reason the binaural beats did not show an effect in Experiment 2. Second, we added a 2-second fixation screen in between each trial. This screen was included for the measurement of pretrial pupil diameter, which was our measure of arousal. Each session included 100 trials. There were 5 practice trials at the beginning of the first session. The order of sessions was counterbalanced such that half of participants received the binaural beat stimulation during the first session of PVT and half received control stimulation during the first session. Participants received the other stimulus during the second session.

\section{Pupillometry}

Pupil diameter and gaze position were recorded binocularly at a $60-\mathrm{Hz}$ sampling rate via a Tobii T1750 eye-tracker. Participants sat $60 \mathrm{~cm}$ from the monitor with their head position fixed in a chinrest. The room was dark, with the only illumination coming from the computer monitor. Participants were seated in a chair that could be adjusted vertically, so participants could sit comfortably in the chinrest without straining or slouching. For each trial, we computed a pretrial pupil diameter measure by averaging measurements over the 2-second fixation window. Then, for each trial, we computed a stimulus-locked change in pupil diameter by subtracting all post-stimulus measurements from a 200-ms wide pre-stimulus baseline window. Measurements were then binned into 100-ms wide, non-overlapping windows for purposes of plotting and statistical analyses. Task-evoked pupillary responses were then averaged within a session for each participant, and compared across sessions and conditions.

\section{Results and Discussion}

Similar to Experiments 1 and 2, we analyzed the behavioral (RT) data using increasingly parameterized linear mixed effects models. In this design there are three fixed within-subject effects: block (1 - 5), session $(1,2)$, and condition (binaural beat, control), and a random effect of subject. Thus, it is a compex design with many nested models of increasingly complexity. The full model contains a main effect of block, a main effect of condition, a main effect of session, a block x condition interaction, a block x session interaction, a condition $\mathrm{x}$ session interaction, and a three-way block $\mathrm{x}$ condition $\mathrm{x}$ session interaction. The coefficients of that full model are shown in Table 5, and the data are plotted in Figure 7. The analyses indicated that there was significant evidence for effect of block, such that RTs increased across blocks, significant evidence for a main effect of session, such that RTs were longer in the second session compared to the first, and a significant block $\mathrm{x}$ session interaction, such that the vigilance decrement was shallower in the second session compared to the first. Finally, there was substantial evidence for a block x condition interaction, such that there was a steeper vigilance decrement in the binaural beat condition. There was also evidence than the binaural beat stimulation had a main effect on RTs, but that this 
was qualified by a large beat $\mathrm{x}$ block interaction. Under the binaural beat stimulation, participants actually demonstrated steeper vigilance decrements, which was the opposite of what we had hypothesized. Finally, the ex-Gaussian parameters also did not differ across conditions - $\mu: t(54)=-0.46, p=0.65, d=0.06 ; \sigma: t(54)=-0.30, p=0.76, d=0.04$; $\tau: t(54)=0.02, p=0.99, d=0.00$. Importantly, none of the effects involving the beat stimulation were consistent with an improvement in performance due to the stimulation in the hypothesized directions.

\section{Pretrial pupil diameter}

Similar to the RT analyses, we examined pretrial pupil diameter as a function of block, condition, and session (see Figure 8). We submitted pretrial pupil diameter to a linear mixed effect model with fixed effects of block, session, and condition, and a random effect of subject. This analysis only provided strong evidence in favor of an effect of block and a three-way block $\mathrm{x}$ session $\mathrm{x}$ condition. But overall, there was strong evidence against the hypothesis that the binaural beat stimulation would either increase or stabilize arousal.

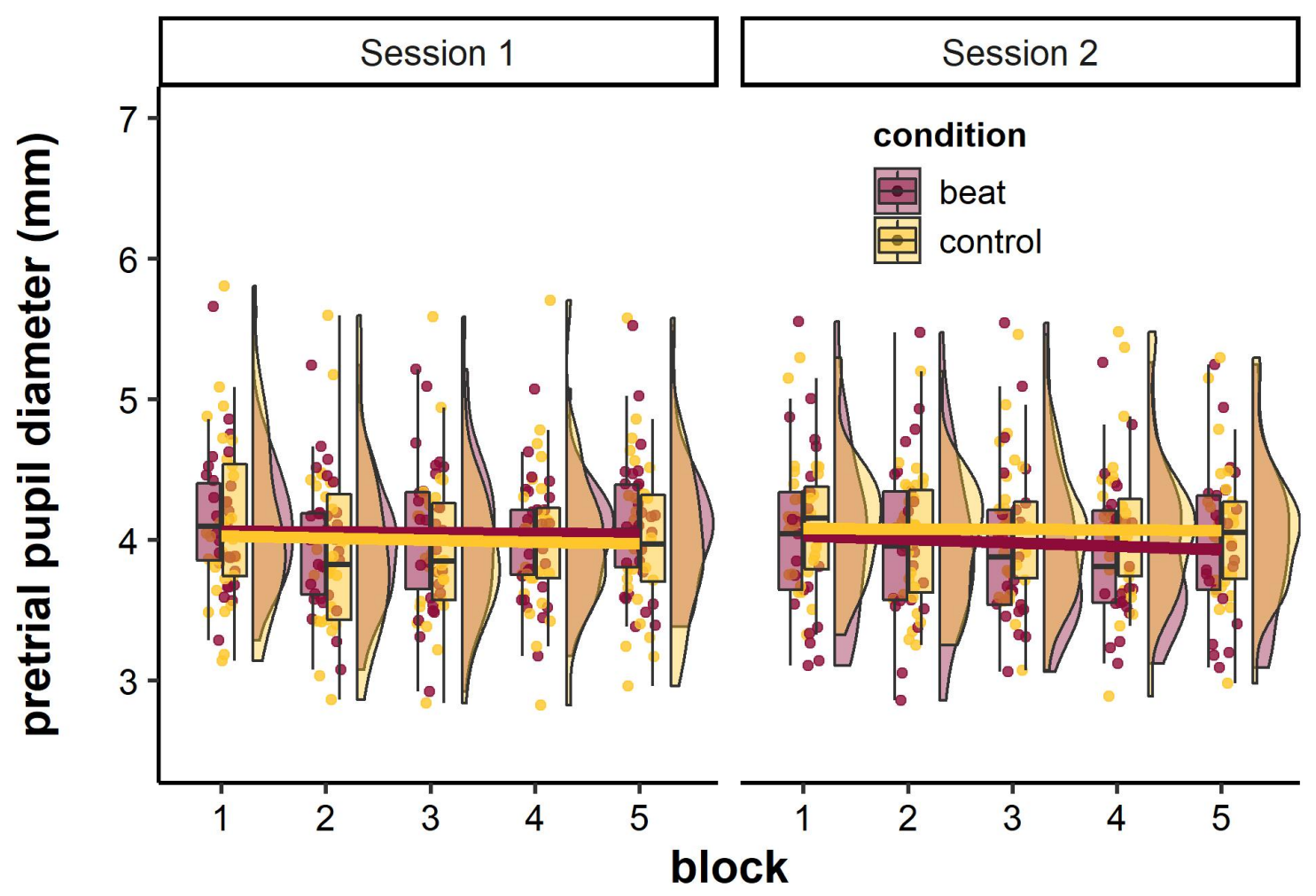

Figure 5. Pretrial pupil diameter by block, condition, and session. There were no significant effects of block, condition, session, or any interactions among these factors in the analyses. Each data point is the mean pretrial pupil diameter within a block for an individual participant. Solid lines represent the fitted effect of block on pretrial pupil diameter for each condition and session. 


\section{Task-evoked pupillary responses (TEPRs)}

In the psychomotor vigilance task, the TEPR usually manifests as a brief, phasic dilation that peaks around 700 - $800 \mathrm{~ms}$ after stimulus onset, and quickly returns to baseline (Massar et al., 2016, 2019; Unsworth \& Robison, 2016, 2017, 2018). Figure 9 shows the average task-evoked response as a function of condition and session. The magnitude and latency of the TEPR is quite similar across conditions and sessions. The only difference appeared to be a quicker return to baseline in Session 2 compared to Session 1. To statistically compare these waveforms, we computed a mean TEPR for each individual for each session by averaging the change from baseline over the full window from 0 - $1200 \mathrm{~ms}$ post stimulus onset. Then we submitted these values to a linear mixed effect model with fixed effects of session and condition and a random effect of subject. The model revealed no significant effects of either session or condition (see Figure 10). TEPRs were smaller in Session 2 because of the quicker return to baseline. This effect did not reach the traditional threshold for significance $(\alpha=.05)$, but there was substantial evidence for it according to the Bayes Factor. There was evidence against a main effect of binaural beat stimulation, and a beat stimulation $\mathrm{x}$ session interaction.

\section{General Discussion}

Researchers do not yet agree on whether sustained attention decrements occur because of depletion of a physiological resource (Warm et al., 2008), shifts in the executive control of attentional focus (Thomson, Besner, \& Smilek, 2015), or shifts in motivation (Kurzban et al., 2013). To address such questions, researchers have attempted to manipulate sustained attention using various experimental manipulations. Given recent research on the use of binaural auditory beat stimulation to modulate cognition, and the fact that such stimulation has been shown to positively affect both memory and attention (Garcia-Argibay et al., 2019), we were interested in whether binaural beats could be an effective way of improving sustained attention. Prior attempts to improve sustained attention have focused on motivational incentives and have provided intriguing results. For example, several studies have demonstrated improvements in sustained attention when participants are rewarded based on performance, given specific goals to attain, or given feedback about their performance (Esterman et al., 2016, 2014; Massar et al., 2016; Robison et al., 2020b). Here, we tried to focus on an exogenous, non-invasive, physiological means of augmenting sustained attention with binaural beats. If indeed binaural beats can augment sustained attention, they could be applied in ways to advance theory and even provide pragmatic solutions for mitigating sustained attention decrements in applied settings.

Intriguingly, in Experiment 1, there was a modest, positive effect of binaural beat stimulation on performance in the psychomotor vigilance task. Participants who heard the beta-frequency $(16 \mathrm{~Hz})$ binaural beat tone demonstrated faster overall RTs compared to a pure tone control condition. However, the effect was rather modest in size $(d=0.35)$, and the Bayes Factor was indiscriminate between inclusion and exclusion of the effect in the model. Participants in the binaural beat condition still showed a vigilance decrement, however, and the magnitude of this effect did not differ across conditions. So we decided to run a follow-up experiment to confirm the presence/absence of the main effect. 




Figure 6. Average task-evoked pupillary responses across participants by session and condition in Experiment 3. Shaded error bars represent $+/$ - one standard error of the mean. 


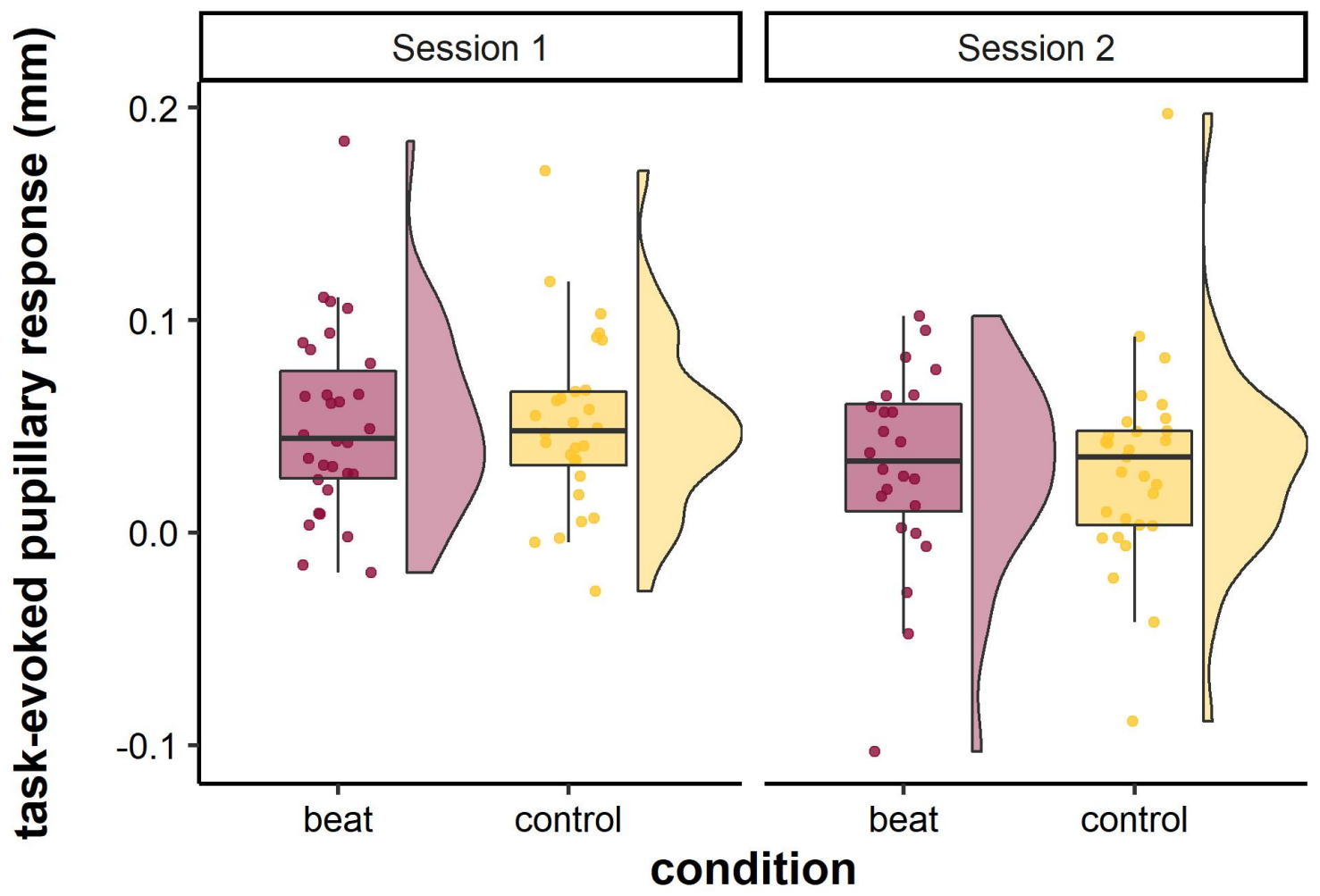

Figure 7. Average task-evoked pupillary response by session and condition in Experiment 3. Each data point is the average task-evoked response in each session for an individual participant. The binaural beat stimulation did not affect the average task-evoked response.

In the follow-up (Experiment 2), we kept most of the task parameters from Experiment 1 the same. However we also added periodic thought probes to the task to measure subjective task-engagement. We did not observe effects of the binaural beat stimulation on either RTs or the thought probe responses. In both the binaural beat and control conditions, RTs increased across blocks and task-engagement decreased. But neither effect differed across conditions. Because Experiments 1 and 2 were so similar, we combined data across these experiments to estimate the magnitude of the effect in the combined data. In this analysis, the effect of the beat stimulation was small $(d=-0.19)$, and the model-comparisons determined that a model without this effect provided a much better fit to the data. However, the analyses also showed some large and unexpected differences in RTs across experiments. Virtually the only differences across Experiments 1 and 2 was the inclusion of thought probes in Experiment 2. To our knowledge, no study has directly compared the influence of thought probes on RTs and vigilance decrements in the psychomotor vigilance task. It is possible that the probes actually exerted an engaging influence on the task - they kept attention more tethered to the task, which led to faster RTs and shallower vigilance decrements. Perhaps this is why there was no effect of binaural beat stimulation in Experiment 2. Prior research on a different sustained attention task (the Sustained Attention to Response Task; SART), has examined the influence of thought probes on task 
performance. These studies demonstrated statistically equivalent task performance when thought probes were included vs. not included (Wiemers \& Redick, 2019), and when thought probes appeared twice as frequently as is typical (Robison et al., 2019). Therefore, we were surprised to observe such differences. The effect in the present study may be spurious, but it deserves additional experimentation in future work.

There are several reasons why we did not find consistent evidence for an effect of binaural beat stimulation on sustained attention in the present study. The first, most straightforward interpretation is that beta-frequency binaural auditory beat stimulation is not powerful enough to augment sustained attention. Or, if it indeed does, the effect is rather small and hard to observe with even large samples. A second reason could be our choice of beat frequency. Some of the studies that have observed attentional effects have used gamma-frequency stimulation (Hommel et al., 2016; Reedijk et al., 2015). However we chose beta-frequency beats because they were successfully used in a vigilance task to improve performance (Lane et al., 1998). A third reason, which we do not believe to be the case, is a lack of power. In a meta-analysis by Garcia-Argibay et al. (2019), the average effect on attention was .58, 95\% CI [.34, -.83]. In Experiment 1, we found an effect of size 0.348, which was within the confidence interval of the meta-analytic estimate of the effect on attention (Garcia-Argibay et al., 2019). We used an a priori power analysis to detect such an effect with a reasonable amount of power (80\%), and we did not replicate the effect. Using the estimated effect size from Garcia-Argibay et al. (2019), our samples would have been able detect effects of that magnitude with $97 \%$ power in Experiment 1, 99.8\% power in Experiment 2, and $99.9 \%$ power in the combined dataset. Therefore, we do not believe our experiments were underpowered to detect effects of theoretical and practical significance.

\section{Limitations and Future Directions}

The present study had several limitations that could be addressed in future research. First, we focused only on one frequency of binaural beats $(16 \mathrm{~Hz})$. In future work, we may need to test other frequencies (e.g., gamma-band), as these have been effective in modulating attention (Hommel et al., 2016; Reedijk et al., 2015). Future research could compare beats of different frequencies, like Lane et al. (1998) did, or various bands against a control stimulus. The other disadvantage of the present study was the fact that our only withinsubjects comparison (Experiment 3) was heavily impacted by the carry over from session

to session. In future work, it may be more advantageous to complete a within-subjects experiment on separate days, which should eliminate any carry over effects.

\section{Conclusion}

Across three experiments that combined objective measures of attention and subjective measures of task-engagement, we did not find any convincing evidence that binaural auditory beat stimulation can augment sustained attention. 


\section{References}

Adam, K. C. S., Mance, I., Fukuda, K., \& Vogel, E. K. (2015). The contribution of attentional lapses to individual differences in visual working memory capacity. Journal of Cognitive Neuroscience, 27, 1601-1616. https://doi.org/10.1162/jocn_a_00811

Aust, F., \& Barth, M. (2018). papaja: Create APA manuscripts with $R$ Markdown. Retrieved from https://github.com/crsh/papaja

Bates, D., Mächler, M., Bolker, B., \& Walker, S. (2015). Fitting linear mixed-effects models using lme4. Journal of Statistical Software, 67, 1-48. https://doi.org/10.18637/jss. v067.i01

Beatty, J. (1982). Task-evoked pupillary responses, processing load, and the structure of processing resources. Psychological Bulletin, 91, 276-292.

Dinges, D. F., \& Powell, J. W. (1985). Microcomputer analyses of performance on a portable, simple visual rt task during sustained operations. Behavior Research Methods, Instruments, \& Computers, 17, 652-655.

Esterman, M., Grosso, M., Liu, G., Mitko, A., Morris, R., \& DeGutis, J. (2016). Anticipation of monetary reward can attenuate the vigilance decrement. PloS One, 11, e0159741.

Esterman, M., Reagan, A., Liu, G., Turner, C., \& DeGutis, J. (2014). Reward reveals dissociable aspects of sustained attention. Journal of Experimental Psychology: General, 143, 2287-2295.

Garcia-Argibay, M., Santed, M. A., \& Reales, J. M. (2019). Efficacy of binaural auditory beats in cognition, anxiety, and pain perception: A meta-analysis. Psychological Research, 83, 357-372.

Hommel, B., Sellaro, R., Fischer, R., Borg, S., \& Colzato, L. S. (2016). High-frequency binaural beats increase cognitive flexibility: Evidence from dual-task crosstalk. Frontiers in Psychology, 7, 1287.

Hopstaken, J. F., Linden, D. van der, Bakker, A. B., \& Kompier, M. A. (2015a). The window of my eyes: Task disengagement and mental fatigue covary with pupil dynamics. Biological Psychology, 110, 100-106.

Hopstaken, J. F., Linden, D. van der, Bakker, A. B., Kompier, M. A., \& Leung, Y. K. (2016). Shifts in attention during mental fatigue: Evidence from subjective, behavioral, physiological, and eye-tracking data. Journal of Experimental Psychology: Human Perception and Performance, 42, 878-889.

Hopstaken, J. F., Van Der Linden, D., Bakker, A. B., \& Kompier, M. A. (2015b). A multifaceted investigation of the link between mental fatigue and task disengagement. Psychophysiology, 52, 305-315.

Kurzban, R., Duckworth, A., Kable, J. W., \& Myers, J. (2013). An opportunity cost model of subjective effort and task performance. Behavioral and Brain Sciences, $36,661-679$. 
Kuznetsova, A., Brockhoff, P. B., \& Christensen, R. H. B. (2017). lmerTest package: Tests in linear mixed effects models. Journal of Statistical Software, 82(13), 1-26. https://doi.org/10.18637/jss.v082.i13

Lakatos, P., Karmos, G., Mehta, A. D., Ulbert, I., \& Schroeder, C. E. (2008). Entrainment of neuronal oscillations as a mechanism of attentional selection. Science, 320, 110113.

Lane, J. D., Kasian, S. J., Owens, J. E., \& Marsh, G. R. (1998). Binaural auditory beats affect vigilance performance and mood. Physiology \& Behavior, 63, 249-252.

Massar, S. A., Lim, J., Sasmita, K., \& Chee, M. W. (2016). Rewards boost sustained attention through higher effort: A value-based decision making approach. Biological Psychology, 120, 21-27. https://doi.org/10.1016/j.biopsycho.2016.07.019

Massar, S. A., Lim, J., Sasmita, K., \& Chee, M. W. (2019). Sleep deprivation increases the costs of attentional effort: Performance, preference and pupil size. Neuropsychologia, 123, $169-177$.

Massidda, D. (2013). Retimes: Reaction time analysis. Retrieved from https://CRAN.Rproject.org $/$ package $=$ retimes

McVay, J. C., \& Kane, M. J. (2012). Drifting from slow to "d'oh!": Working memory capacity and mind wandering predict extreme reaction times and executive control errors. Journal of Experimental Psychology: Learning, Memory, and Cognition, 38, $525-549$.

Morey, R. D., \& Rouder, J. N. (2018). BayesFactor: Computation of bayes factors for common designs. Retrieved from https://CRAN.R-project.org/package=BayesFactor

Reedijk, S. A., Bolders, A., Colzato, L. S., \& Hommel, B. (2015). Eliminating the attentional blink through binaural beats: A case for tailored cognitive enhancement. Frontiers in Psychiatry, 6, 82.

Robison, M. K. (2018). Regulating mind-wandering and sustained attention with goalsetting, feedback, and incentives. Doctoral Dissertation. Retrieved from http://hdl. handle.net/1794/23712

Robison, M. K., Ellis, D. M., Pitaes, M. M., Karoly, P., \& Brewer, G. A. (2020a). Acute pain impairs sutained attention. PsyArXiv. Retrieved from https://psyarxiv.com/w87rj/

Robison, M. K., Miller, A. L., \& Unsworth, N. (2019). Examining the effects of probe frequency, response options, and framing within the thought-probe method. Behavior Research Methods, 51, 398-408.

Robison, M. K., \& Unsworth, N. (2019). Pupillometry tracks fluctuations in working memory performance. Attention, Perception, 6 Psychophysics, 81, 407-419. https://doi.org/10.3758/s13414-018-1618-4

Robison, M. K., Unsworth, N., \& Brewer, G. A. (2020b). Examining the effects of goalsetting, feedback, and incentives on sustained attention. Manuscript Under Review.

Solcà, M., Mottaz, A., \& Guggisberg, A. G. (2016). Binaural beats increase interhemispheric alpha-band coherence between auditory cortices. Hearing Research, 332, 233-237. 
Thomson, D. R., Besner, D., \& Smilek, D. (2015). A resource-control account of sustained attention: Evidence from mind-wandering and vigilance paradigms. Perspectives on Psychological Science, 10.

Thomson, D. R., Seli, P., Besner, D., \& Smilek, D. (2014). On the link between mind wandering and task performance over time. Consciousness and Cognition, 27, 1426.

Unsworth, N., \& Robison, M. K. (2016). Pupillary correlates of lapses of sustained attention. Cognitive, Affective, $\mathcal{E}$ Behavioral Neuroscience, 16, 601-615. https://doi.org/10. 3758/s13415-016-0417-4

Unsworth, N., \& Robison, M. K. (2017). The importance of arousal for variation in working memory capacity and attention control: A latent variable pupillometry study. Journal of Experimental Psychology: Learning, Memory, and Cognition, 43, 1962-1987. https://doi.org/10.1037/xlm0000421

Unsworth, N., \& Robison, M. K. (2018). Tracking arousal state and mind wandering with pupillometry. Cognitive, Affective, \& Behavioral Neuroscience, 18, 638-664. https://doi.org/10.3758/s13415-018-0594-4

Unsworth, N., \& Robison, M. K. (2020). Working memory capacity and sustained attention: A cognitive-energetic perspective. Journal of Experimental Psychology: Learning, Memory, and Cognition, 46, 77-103.

Warm, J. S., Parasuraman, R., \& Matthews, G. (2008). Vigilance requires hard mental work and is stressful. Human Factors, 50, 433-441.

Weinstein, Y. (2018). Mind-wandering, how do i measure thee with probes? Let me count the ways. Behavior Research Methods, 50, 642-661.

Wickham, H. (2017). Tidyverse: Easily install and load the 'tidyverse'. Retrieved from https://CRAN.R-project.org/package=tidyverse

Wiemers, E. A., \& Redick, T. S. (2019). The influence of thought probes on performance: Does the mind wander more if you ask it? Psychonomic Bulletin $\&$ Review, 26, 367-373. 